\title{
Area of Brownian Motion with Generatingfunctionology
}

\author{
Michel Nguyên Thê ${ }^{1,2}$ \\ ${ }^{1}$ Laboratoire d'Informatique de l'X, École Polytechnique, 91128 Palaiseau Cedex, France \\ ${ }^{2}$ Institut Gaspard Monge, Laboratoire d'Informatique, 77454 Marne-la-Vallée Cedex 2, France \\ mnguyendix.polytechnique.fr
}

This paper gives a survey of the limit distributions of the areas of different types of random walks, namely Dyck paths, bilateral Dyck paths, meanders, and Bernoulli random walks, using the technology of generating functions only.

Keywords: Dyck path, Bernoulli random walk, Brownian motion, generating functions, weak convergence of stochastic processes.

\section{Introduction}

The distribution of the (absolute) area of Brownian motion was first studied by Mark Kac [Kac (1949)], using a formula since known as the Feynman-Kac formula [Kac (1949); Ito and McKean (1965)], which allows us to find the double Laplace transform of the distribution of the integral of a functional of Brownian motion.

The area functional has received intensive attention for different types of Brownian motion, such as standard Brownian motion, Brownian bridges, excursions and meanders [Cifarelli (1975); Shepp (1982); Takács (1993); Perman and Wellner (1995); Jeanblanc et al. (1997); Tolmatz (2000); Tolmatz (2001); ...].

These different types of motion have discrete counterparts, that are respectively Bernoulli walks, bilateral Dyck paths, Dyck paths [Stanley (1999)], and (discrete) meanders. All paths considered in this paper start from $(0,0)$ and only have +1 and -1 steps. A bilateral Dyck path is a Bernoulli random walk starting from $(0,0)$ and ending in $(0,0)$. A Dyck path is a bilateral Dyck path conditioned to stay over the $x$-axis. A meander is a Bernoulli random walk conditioned to stay over the $x$-axis.

As their lengths tend to infinity, we have weak convergence of the functional of these paths (conditioned by size) to their continuous counterpart, and convergence of the moments [Drmota (2003)].

Studying the distribution of a Brownian motion hence amounts to studying the limit distribution of its discrete counterpart. This was first done in the discrete world by Takács [Takács (1991a)] for Dyck paths. As Dyck paths are building blocks for bilateral Dyck paths, it is easy to deduce the limit distribution of the area of bilateral Dyck paths from the one of Dyck paths. It is interesting to notice, as commented in [Nguy^en Th^e (2003)], that it is the other way round in the continuous world, where the area of excursions is deduced from the area of Brownian bridges [Louchard (1984)]. In the same vein, while in the continuous world the area of Brownian motion can be computed directly, we first have to study the area 
of meanders in the context of generating functions before finding the distribution of the area of Brownian motion.

Using the Feynman-Kac formula, computing the distribution of signed areas of Brownian motion and of Brownian bridges is straightforward [Perman and Wellner (1995)]. This is also the case using generatingfunctionology (the technology of generating functions).

\section{Generating functions}

\subsection{Functional equations}

Let $D, F, P$ and $B$ denote the generating functions associated with Dyck paths, meanders, bilateral Dyck paths and Bernoulli random walks (with steps -1 and +1 ) respectively, where $z$ marks the size of the walks, and $u$ its area, understood as the sum of the heights. For example, if $\mathcal{D}$ is the set of all Dyck paths, then

$$
D(z, u)=\sum_{d \in \mathcal{D}} z^{|d|} u^{\text {area }(d)} .
$$

A classical Dyck path will be called positive and its reflection across the $x$-axis will be called negative. In the same way, we define the notions of positive and negative meanders. We have

\section{Proposition 1.}

$$
\begin{aligned}
D(z, u) & =1+z^{2} u D(z, u) D(z u, u), \\
F(z, u) & =D(z, u)+u z D(z, u) F(z u, u), \\
P(z, u) & =\frac{1}{1-2 u z^{2} D(z u, u)}, \\
B(z, u) & =P(z, u)(2 z u F(z u, u)+1) .
\end{aligned}
$$

Proof. A Dyck path is either an empty Dyck path, enumerated by $1 \cdot z^{0}=1$, or a Dyck path, enumerated by $D(z, u)$, followed by a nonempty Dyck path, enumerated by $z^{2} u D(z u, u)$. (See [Takács (1991a)] for the details of computation).

A meander is either a Dyck path, that is a meander ending at zero, enumerated by $D(z, u)$, or a Dyck path, enumerated by $D(z, u)$, followed by a meander not ending at zero, enumerated by $u z F(z u, u)$.

A bilateral Dyck path is a sequence of nonempty positive or negative Dyck paths.

A Bernoulli walk is either ending at zero, in which case it is a bilateral Dyck path, enumerated by $P(z, u)$, or not ending at zero, in which case it is a bilateral Dyck path, enumerated by $P(z, u)$, followed by a positive or a negative meander, enumerated by $2 z u F(z u, u)$.

\subsection{The method of moments}

As we said in the introduction, we are interested in the limit distribution of the area of certain types of discrete random walks, when theirs sizes tend to infinity. Weak convergence will hold, but also convergences of moments of any order, either thanks to Drmota's work, or by Fréchet-Shohat moment convergence theorem, given Carleman's condition [Feller (1971)]. Using singularity analysis on generating functions, we will determine recurrence relations on the moments of different orders of the limit distribution. They 
allow to check Carleman's condition, but also to show that the limit distribution admits a Laplace transform which can be expanded around 0 (we will show how to proceed for Dyck paths, and leave the other cases to the reader). After some analysis, we can identify the limit distribution by its Laplace transform (which is determined by a kind of double Laplace transform of the distribution).

\section{Dyck paths and bilateral Dyck paths}

We recall here results concerning the limit distribution of the area of Dyck paths and bilateral Dyck paths which have already been shown with the use of generating functions. We outline the proofs, not only because the proofs in the following section will follow the same methodology, but also to introduce intermediate computations that will turn out to be useful in the next section.

The reader interested in complete proofs can either look at Takács's papers, or look at the more detailed proofs of lemma 1 and theorem 4.

If $B_{s}$ is a Brownian motion, the scaling property $B_{s} \stackrel{\mathcal{D}}{\equiv} \sqrt{\ell} B_{s / \ell}$, for all positive $\ell$, which is valid for meanders, bridges and excursions $(\ell$ is then an independent random variable equal to the duration of the motion in the last two cases), induces

$$
\int_{0}^{t}\left|B_{s}\right| d s \stackrel{\mathcal{D}}{=} t^{3 / 2} \int_{0}^{1}\left|B_{s}\right| d s
$$

with similar formulas for meanders, bridges and excursions, conditioned to have duration 1 on the righthand side. Hence, as far as Dyck paths and bilateral Dyck paths of length $2 n$ are concerned, it is natural to normalize their area by $(2 n)^{3 / 2}$. It would be easy to check (see [Takács (1991a)] or the computations of the first two moments below) that the variance of the area of Dyck paths and bilateral Dyck paths is indeed of order $\sigma^{2} n^{3}$ with $\sigma^{2}>0$, though $\sigma \neq 2$.

Theorem 1. Let $\phi_{D}$ be the Laplace transform of the area of excursions conditioned to have duration 1. Then it satisfies

$$
\int_{0}^{\infty}\left(e^{-u s}-1\right) \phi_{D}\left(\sqrt{2} s^{3 / 2}\right) \frac{d s}{s^{3 / 2}}=2 \sqrt{\pi}\left(\frac{A i^{\prime}(u)}{A i(u)}-\frac{A i^{\prime}(0)}{A i(0)}\right) .
$$

Proof. We outline Takács's proof [Takács (1993)].

Differentiating (2) $r$ times with respect to $u$ and setting $u=1$, one can show by induction that there exists for each $r$ a non-null constant $K_{r}$ such that

$$
\frac{D_{u^{r}}(z, 1)}{r !} \underset{z \rightarrow \frac{1}{2}}{\sim} \frac{2^{r+2} K_{r}}{\left(1-4 z^{2}\right)^{\frac{3 r-1}{2}}}
$$

(In $D_{u^{r}}$, the notation $u^{r}$ represents the word $u u \cdots u$, where $u$ appears $r$ times, so that $D_{u^{r}}$ represents the function $(z, u) \mapsto D(z, u)$ differentiated $r$ times with respect to $u$. The notation $\sim$ means that the left hand side and the right hand side are equivalent as $z$ tends to $1 / 2)$. In addition, the sequence $\left(K_{r}\right)$ satisfies for $r \geq 1$ the recurrence

$$
K_{r}=\sum_{i=1}^{r-1} K_{i} K_{r-i}-K_{0} K_{r-1}(3 r-4) / 2
$$


with $K_{0}=-1 / 2$. The series $y(z)=\sum K_{r}(-z)^{r}$ is then the solution of the equation

$$
3 z^{2} y^{\prime}(z)-z y(z)=4 y^{2}(z)-1,
$$

which (given $y(0)=-1 / 2$ ) can be solved to yield

$$
y(z)=A i^{\prime}\left(z^{-2 / 3}\right) z^{1 / 3} /\left[2 A i\left(z^{-2 / 3}\right)\right],
$$

where $A i$ is the Airy function, which is the solution of $w^{\prime \prime}=x w$ such that $\lim _{x \rightarrow \infty} w(x)=0$ [p. 446 of Abramowitz and Stegun (1973)]. If $M_{r}^{D}$ is the moment of order $r$ of the limit distribution of the area of Dyck paths normalized by $(\sqrt{2 n})^{3}$, then singularity analysis [Flajolet and Sedgewick (1993)] gives

$$
M_{r}^{D}=\lim _{n \rightarrow \infty} \frac{\left[z^{2 n}\right] D_{u^{r}}(z, 1)}{(\sqrt{2 n})^{3 r}\left[z^{2 n}\right] D(z, 1)}=\lim _{n \rightarrow \infty} \frac{\left[z^{2 n}\right] \frac{2^{r+2} K_{r}}{\left(1-4 z^{2}\right)^{\frac{3 r-1}{2}}}}{(\sqrt{2 n})^{3 r}\left[z^{2 n}\right]\left(-2 \sqrt{1-4 z^{2}}\right)}=\frac{4 \sqrt{\pi} K_{r} r !}{\Gamma\left(\frac{3 r-1}{2}\right) 2^{\frac{r}{2}}} .
$$

From recursion (9), it is easy to deduce that there exists a constant $\rho>0$ such that

$$
K_{r} / r !=\underset{r \rightarrow \infty}{O}\left(\rho^{-r}\right)
$$

One can for example introduce the series $g(z)=\sum g_{r} z^{r}$ with $g_{0}=K_{0}, g_{1}=K_{1}$, and for $r \geq 2$,

$$
g_{r}=\sum_{j=1}^{r-1} g_{j} g_{r-j}+\frac{3}{4} g_{r-1}
$$

so that for all $r, K_{r} \leq r ! g_{r}$. Then one can choose $\rho$ to be equal to the radius of convergence of $g$, which is positive. Notice that it is possible to find an equivalent for $\left(K_{r}\right)$ as $r$ tends to infinity [Takács (1991b)], but it requires much more computations, involving the asymptotic expansion of the Airy function.

From (13) we can deduce an upper bound for $\left(M_{r}\right)$, and check that $\left(M_{r}\right)$ satisfies Carleman's condition, so that we have both weak convergence and convergence of moments of any order. We also deduce that the Laplace transform of the limit distribution

$$
\phi_{D}(x)=\sum \frac{M_{r}^{D}}{r !}(-x)^{r}
$$

admits a series expansion around 0 . It is then easy to obtain (7) after integration by parts.

Theorem 1 was first established by Louchard (1984), starting from the known double Laplace transform of the area of bridges and using the following result:

Theorem 2 (Louchard). Let $W_{t}$ denote the Brownian motion, and $e_{t}$ the Brownian excursion. If $h \geq 0$ is a symmetric piecewise continuous function, if

$$
g(\alpha):=\int_{0}^{\infty} e^{-\alpha t} \mathbb{E}_{0}\left[\exp \left(-\int_{0}^{t} h\left[W_{s}\right] d s\right) \mid W_{t}=0\right] \frac{d t}{\sqrt{2 \pi t}}
$$

and if $L$ is the duration of excursion $e$, then

$$
\int_{0}^{\infty}(\exp (-\alpha u)-1) \mathbb{E}\left[\exp \left(-\int_{0}^{u} h\left(e_{s}\right) d s\right) \mid L=u\right] \frac{d u}{\sqrt{2 \pi u^{3}}}=-g^{-1}(\alpha)+\lim _{\alpha \rightarrow 0} g^{-1}(\alpha) .
$$


In the context of generating functions, we will use a kind of converse of this theorem (or rather give another proof of it for the functional of interest), to determine the distribution of the area of bridges as a function of the distribution of the area of excursions. We then recover a result established by Shepp [Shepp (1982)].

Theorem 3 (Shepp). Let $\phi_{P}$ be the Laplace transform of the distribution of absolute area of Brownian bridges conditioned to have duration 1. Then it satisfies

$$
\int_{0}^{\infty} e^{-u s} \phi_{P}\left(\sqrt{2} s^{3 / 2}\right) s^{-1 / 2} d s=-\sqrt{\pi} \frac{A i(u)}{A i^{\prime}(u)} .
$$

Proof. We proceed as in [Nguy^en Th^e (2003)]. Differentiating

$$
\left(1-2 u z^{2} D(z u, u)\right) P(z, u)=1
$$

$r$ times with respect to $u$ and setting $u=1$, one can show by induction that there exists a sequence $\left(Q_{r}\right)$ of positive numbers such that

$$
\frac{1}{r !} P_{u^{r}}(z, 1) \underset{z \rightarrow \frac{1}{2}}{\sim} \frac{2^{r} Q_{r}}{\left(\sqrt{1-4 z^{2}}\right)^{3 r+1}}
$$

This sequence is given by

$$
Q_{0}=1 \quad \text { and } \quad \forall r \geq 1, Q_{r}=2 \sum_{j=1}^{r} K_{j} Q_{r-j}
$$

The function $q(z)=\sum_{r \geq 0} Q_{r}(-z)^{r}$ satisfies

$$
q(z)=-\frac{1}{2 y(z)}=-\frac{A i\left(z^{-2 / 3}\right)}{A i^{\prime}\left(z^{-2 / 3}\right) z^{1 / 3}} .
$$

Defining $\left(M_{r}^{P}\right)$ such that $\phi_{P}(x)=\sum \frac{M_{r}^{P}}{r !}(-x)^{r}$, singularity analysis gives

$$
M_{r}^{P}=\lim _{n \rightarrow \infty} \frac{\left[z^{2 n}\right] P_{u^{r}}(z, 1)}{(2 n)^{\frac{3 r}{2}}\left[z^{2 n}\right] P(z, 1)}=\sqrt{\pi} \frac{Q_{r} r !}{2^{r / 2} \Gamma\left(\frac{3 r+1}{2}\right)},
$$

and it is then easy to establish formula (16).

\section{Meander and Bernoulli random walk}

In this section we recover the distribution of the areas of meanders and Bernoulli random walks. As it has apparently not been done before using generating functions, we will give computations with more details than in the previous section. 
Lemma 1. For all $r$, there exist non-null constants $a_{r}^{D}, a_{r}^{F}, a_{r}^{P}$, and $a_{r}^{B}$, such that,

$$
\begin{array}{ccc}
\frac{D_{u^{r}}(z, 1)}{r !} & \underset{z \rightarrow \frac{1}{2}}{\sim} \frac{a_{r}^{D}}{(1-2 z)^{\frac{3 r-1}{2}}}, \\
\frac{F_{u^{r}}(z, 1)}{r !} \underset{z \rightarrow \frac{1}{2}}{\sim} \frac{a_{r}^{F}}{(1-2 z)^{\frac{3 r+1}{2}}}, \\
\frac{P_{u^{r}}(z, 1)}{r !} \underset{z \rightarrow \frac{1}{2}}{\sim} \frac{a_{r}^{P}}{(1-2 z)^{\frac{3 r+1}{2}}}, \\
\frac{B_{u^{r}}(z, 1)}{r !} \underset{z \rightarrow \frac{1}{2}}{\sim} \frac{a_{r}^{B}}{(1-2 z)^{\frac{3 r+2}{2}}},
\end{array}
$$

where the sequence $\left(a_{r}^{F}\right)_{r \geq 0}$ is given by the initial condition $a_{0}^{F}=\sqrt{2}$ and the recurrence

$$
\sqrt{2} a_{r}^{F}=\frac{1}{4}(3 r-2) a_{r-1}^{F}+\frac{1}{2} \sum_{j=0}^{r-1} a_{r-j}^{D} a_{j}^{F} \quad(r \geq 1) .
$$

In addition, for all $r \geq 0$, the sequence $\left(a_{r}^{B}\right)_{r \geq 0}$ satisfies

$$
a_{r}^{B}=\sum_{j=0}^{r} a_{r-j}^{P} a_{j}^{F} .
$$

Proof. According to the previous results, $a_{r}^{D}$ and $a_{r}^{P}$ obviously exist and are equal to $2^{r+2} K_{r}$ and $2^{-\frac{r+1}{2}} Q_{r}$ respectively. This settles (22) and (24).

Let us now show (23). We have

$$
F(z, 1)=\frac{-1+\frac{1+2 z}{1-2 z}}{2 z} \underset{z \rightarrow \frac{1}{2}}{\sim} \frac{\sqrt{2}}{\sqrt{1-2 z}}
$$

so that (23) is true for $r=0$.

After differentiating equation (3) $r$ times, we obtain

$$
F_{u^{r}}=D_{u^{r}}+z \sum_{j=0}^{r}\left(\begin{array}{l}
r \\
j
\end{array}\right) D_{u^{r-j}}(u F(z u, u))^{(j)}
$$

with

$$
(u F(z u, u))^{(j)}=u\left(F_{u^{j}}+j z F_{z u^{j-1}}+\cdots\right)+1 \cdot(F(z u, u))^{(j-1)} .
$$

Hence for $u=1$

$$
(1-z D) F_{u^{r}}=r z^{2} D F_{z u^{r-1}}+z \sum_{j=0}^{r-1}\left(\begin{array}{l}
r \\
j
\end{array}\right) D_{u^{r-j}} F_{u^{j}}+\cdots
$$

where the trailing terms are negligible compared to the given terms when $z$ tends towards 2 . 
Set $R=(\sqrt{1-2 z})^{-1}$. Then $D_{u^{j}}$ and $F_{u^{j}}$ have dominant terms in $R$ of degrees $3(r-j)-1$ and $3 j+1$ respectively, so that $D_{u^{j}} F_{u^{r-j}}$ is of degree $3 r$. In addition, the asymptotics for $z$ tending towards $\frac{1}{2}$

$$
\frac{F_{u^{r-1}}}{(r-1) !} \sim \frac{a_{r-1}^{F}}{(1-2 z)^{\frac{3(r-1)+1}{2}}}=\frac{a_{r-1}^{F}}{(1-2 z)^{\frac{3 r-2}{2}}}
$$

implies

$$
\frac{F_{z u^{r-1}}}{(r-1) !} \sim \frac{(3 r-2) a_{r-1}^{F}}{(1-2 z)^{\frac{3 r}{2}}}
$$

so that $F_{z u^{r-1}}$ is of degree $3 r$. As we have

$$
1-z D \underset{z \rightarrow \frac{1}{2}}{\sim} \sqrt{2} \sqrt{1-2 z}
$$

we deduce that $(1-z D) F_{u^{r}}$ is of degree $3 r$, so that $F_{u^{r}}$ is of degree $3 r+1$ and (23) is proved.

We have $\left(z^{2} D\right)(2)=\frac{1}{2}$ hence, by identifying the coefficients of the monomial $R^{3 n}$, we obtain

$$
\sqrt{2} a_{r}^{F} r !=\frac{1}{2} r(r-1) !(3 r-2) a_{r-1}^{F}+\frac{1}{2} \sum_{j=0}^{r-1}\left(\begin{array}{l}
r \\
j
\end{array}\right)(r-j) ! a_{r-j}^{D} j ! a_{j}^{F},
$$

which, divided by $r$ !, becomes (26).

We show (25) and (27) in the same way. Differentiating (5) $r$ times, we obtain

$$
B_{u^{r}}(z, 1)=\sum_{j=0}^{r}\left(\begin{array}{l}
r \\
j
\end{array}\right) P_{u^{r-j}}(z, 1) F_{u^{j}}(z, 1)+\cdots
$$

which gives (25) with $\left(a_{r}^{B}\right)$ satisfying (27).

We can now determine the double Laplace transforms of the distributions of the areas of meanders and Brownian motion. Notice that the length $n$ of discrete meanders and Bernoulli random walks can be as well even or odd, so that we will normalize the random variable by $n^{3 r / 2}$.

Theorem 4. Let $\phi_{F}$ be the Laplace transform of the distribution of the absolute area of Brownian meanders of duration 1. Then it satisfies

$$
\int_{0}^{\infty} e^{-u s} \phi_{F}\left(\sqrt{2 s^{3}}\right) s^{-1 / 2} d s=-\sqrt{\pi} \frac{3 \int_{0}^{u} A i(t) d t-1}{3 A i(u)} .
$$

Proof. Since $D \sim-\sqrt{2} R$, we have $a_{0}^{D}=-\sqrt{2}$, so that (26) can be rewritten

$$
\frac{3}{2}(r-1) a_{r-1}^{F}+\frac{1}{2} a_{r-1}^{F}+\frac{1}{2} \sum_{j=0}^{r} a_{r-j}^{D} a_{j}^{F}=0 .
$$

If we multiply (38) by $(-x)^{r}$ and then sum it from $r=1$ to infinity, we get

$$
\frac{3}{2} x^{2} A_{F}^{\prime}-2 x A_{F}+\frac{1}{2}\left(A_{F} A_{D}-a_{0}^{F} a_{0}^{D}\right)=0
$$


where

$$
A_{F}(x)=\sum_{j=0}^{r} a_{r}^{F}(-x)^{r} \quad \text { and } \quad A_{D}(x)=\sum_{j=0}^{r} a_{r}^{D}(-x)^{r} .
$$

As we have $a_{0}^{F}=\sqrt{2}$, (39) can be rewritten

$$
-\frac{3}{2} x^{2} A_{F}^{\prime}+\left(\frac{A_{D}}{2}-\frac{1}{2} x\right) A_{F}+2=0,
$$

and, replacing $x$ with $\frac{1}{2 z^{3 / 2}}$, we obtain

$$
-\frac{3}{2} \frac{1}{4 z^{3}} A_{F}^{\prime}\left(\frac{1}{2 z^{3 / 2}}\right)+\left(\frac{1}{2} A_{D}\left(\frac{1}{2 z^{3 / 2}}\right)-\frac{1}{4 z^{3 / 2}}\right) A_{F}\left(\frac{1}{2 z^{3 / 2}}\right)+2=0 .
$$

Set $f(z)=A_{F}\left(\frac{\sqrt{2}}{z^{3 / 2}}\right)$. It satisfies

$$
f^{\prime}(z)=-\frac{3 \sqrt{2}}{z^{3 / 2}} A_{F}^{\prime}\left(\frac{\sqrt{2}}{z^{3 / 2}}\right) .
$$

We already know

$$
A_{D}(x)=4 \sqrt{2} y\left(\frac{x}{\sqrt{2}}\right)=4 \sqrt{2} \frac{A i^{\prime}\left(\left(\frac{x}{\sqrt{2}}\right)^{-2 / 3}\right)}{2 A i\left(\left(\frac{x}{\sqrt{2}}\right)^{-2 / 3}\right)}
$$

so that

$$
A_{D}\left(\frac{\sqrt{2}}{z^{3 / 2}}\right)=2 \sqrt{2} \frac{A i^{\prime}(z) z^{-1 / 2}}{A i(z)}
$$

and

$$
f^{\prime}(z)=\left(\frac{1}{2 z}-\frac{A i^{\prime}(z)}{A i(z)}\right) f(z)-\sqrt{2 z} .
$$

Using the method of variation of parameters, and given the initial condition $f(\infty)=\sqrt{2}$ and the asymptotics 10.4.59 and 10.4.82 of [Abramowitz and Stegun (1973)] for large $x$,

$$
A i(x) \sim \frac{1}{2} \pi^{-1 / 2} x^{-1 / 4} e^{-\frac{2}{3} x^{3 / 2}},
$$

and

$$
\int_{0}^{x} A i(t) d t \sim \frac{1}{3}-\frac{1}{2} \pi^{-1 / 2} x^{-3 / 4} \exp \left(-\frac{2}{3} x^{3 / 2}\right)
$$

we easily find that

$$
f(z)=-\sqrt{2 z} \frac{3 \int_{0}^{z} A i(u) d u-1}{3 A i(z)} .
$$


Singularity analysis applied to $F_{u^{r}}(z, 1)$ gives

$$
M_{r}^{F}=\lim _{n \rightarrow \infty} \frac{\left[z^{n}\right] F_{u^{r}}(z, 1)}{n^{\frac{3 r}{2}}\left[z^{n}\right] F(z, 1)}=\frac{\sqrt{\pi} r ! a_{r}^{F}}{\sqrt{2} \Gamma\left(\frac{3 r+1}{2}\right)} .
$$

Finally

$$
\begin{aligned}
\int_{0}^{\infty} e^{-u s} \phi_{F}\left(\sqrt{2 s^{3}}\right) s^{-1 / 2} d s & =\int_{0}^{\infty} \sum_{r \geq 0} \frac{M_{r}^{F}}{r !}\left(-\sqrt{2} s^{3 / 2}\right)^{r} s^{-1 / 2} e^{-u s} d s \\
& =\frac{\pi}{2} \sum_{r \geq 0} \int_{0}^{\infty} \frac{a_{r}^{F}}{\left(\frac{3 r+1}{2}\right)}\left(-\sqrt{2} s^{3 / 2}\right)^{r} s^{-1 / 2} e^{-u s} d s \\
& =\frac{\pi}{2} \sum_{r \geq 0} a_{r}^{F}\left(\frac{\sqrt{2}}{u}\right)^{(3 r+1) / 2}=\sqrt{\frac{\pi}{2 u}} A_{F}\left(\frac{\sqrt{2}}{u^{3 / 2}}\right) \\
& =-\sqrt{\pi} \frac{3 \int_{0}^{u} A i(t) d t-1}{3 A i(u)} .
\end{aligned}
$$

Theorem 5. Let $\phi_{B}$ be the Laplace transform of the distribution of the absolute area of Brownian motions of duration 1. Then

$$
\int_{0}^{\infty} e^{-z t} \phi_{B}\left(\sqrt{2 t^{3}}\right) d t=\frac{3 \int_{0}^{z} A i(u) d u-1}{3 A i^{\prime}(z)}
$$

Proof. Set $A_{P}(x)=\sum_{n \geq 0} a_{n}^{P}(-x)^{n}$, which is given by

$$
A_{P}(x)=\frac{1}{\sqrt{2}} g\left(\frac{x}{\sqrt{2}}\right)=-\frac{1}{\sqrt{2}} \frac{2 A i\left(\left(\frac{x}{\sqrt{2}}\right)^{-2 / 3}\right)}{A i^{\prime}\left(\left(\frac{x}{\sqrt{2}}\right)^{-2 / 3}\right)}=-\frac{1}{\sqrt{2}} \frac{A i(z) z^{1 / 2}}{A i^{\prime}(z)}
$$

From (27) we deduce that

$$
A_{B}(x)=A_{P}(x) A_{B}(x)
$$

so that

$$
\begin{aligned}
A_{B}\left(\frac{\sqrt{2}}{z^{3 / 2}}\right) & =A_{P}\left(\frac{\sqrt{2}}{z^{3 / 2}}\right) A_{F}\left(\frac{\sqrt{2}}{z^{3 / 2}}\right)=\left(-\frac{1}{\sqrt{2}} \frac{A i(z) z^{1 / 2}}{A i^{\prime}(z)}\right) \cdot\left(\sqrt{2 z} \frac{3 \int_{0}^{z} A i(u) d u-1}{3 A i(z)}\right) \\
& =z \frac{3 \int_{0}^{z} A i(u) d u-1}{3 A i^{\prime}(z)}
\end{aligned}
$$

which can be written as (55). 


\section{Signed areas}

In [Perman and Wellner (1995)] is the following theorem, which provides the joint distribution of the area of Brownian motion over and under the $x$-axis, using the Feynman-Kac formula.

Theorem 6. Define

$$
x^{+}=\left\{\begin{array}{ll}
x & \text { if } x \geq 0, \\
0 & \text { otherwise, }
\end{array} \quad \text { and } \quad x^{-}=\left\{\begin{array}{cl}
-x & \text { if } x \leq 0, \\
0 & \text { otherwise. }
\end{array}\right.\right.
$$

Let $V$ be the function $x \mapsto \beta x^{+}+\gamma x^{-}$. Then for $\alpha, \beta, \gamma>0$ we have

$$
\begin{aligned}
& \int_{0}^{\infty} e^{-\alpha t} \mathbb{E}\left[e^{\left.-t^{3 / 2} \int_{0}^{1} V\left(b_{s}\right) d s\right]} \frac{d t}{\sqrt{2 \pi t}}\right. \\
& =-\frac{2 A i\left((2 \gamma)^{1 / 3} \alpha / \gamma\right) A i\left((2 \beta)^{1 / 3} \gamma / \alpha\right)}{(2 \gamma)^{1 / 3} A i^{\prime}\left((2 \gamma)^{1 / 3} \alpha / \gamma\right) A i\left((2 \beta)^{1 / 3} \alpha / \beta\right)+(2 \beta)^{1 / 3} A i^{\prime}\left((2 \beta)^{1 / 3} \alpha / \beta\right) A i\left((2 \gamma)^{1 / 3} \alpha / \gamma\right)}
\end{aligned}
$$

and, if $W_{t}$ denotes the standard Brownian motion,

$$
\begin{aligned}
& \int_{0}^{\infty} e^{-\alpha t} \mathbb{E}_{0}\left[e^{-\int_{0}^{t} V\left(W_{s}\right) d s}\right] d t \\
& =-\frac{2 A i\left((2 \beta)^{1 / 3} \alpha / \beta\right) \int_{0}^{\infty} A i\left((2 \gamma)^{1 / 3}(x+\alpha / \gamma)\right) d x}{(2 \gamma)^{1 / 3} A i\left((2 \gamma)^{1 / 3} \alpha / \gamma\right) A i\left((2 \beta)^{1 / 3} \alpha / \beta\right)+(2 \beta)^{1 / 3} A i\left((2 \beta)^{1 / 3} \alpha / \beta\right) A i\left((2 \gamma)^{1 / 3} \alpha / \gamma\right)} \\
& \quad-\frac{2 A i\left((2 \gamma)^{1 / 3} \alpha / \gamma\right) \int_{0}^{\infty} A i\left((2 \beta)^{1 / 3}(x+\alpha / \beta)\right) d x}{(2 \gamma)^{1 / 3} A i\left((2 \gamma)^{1 / 3} \alpha / \gamma\right) A i\left((2 \beta)^{1 / 3} \alpha / \beta\right)+(2 \beta)^{1 / 3} A i\left((2 \beta)^{1 / 3} \alpha / \beta\right) A i\left((2 \gamma)^{1 / 3} \alpha / \gamma\right)}
\end{aligned}
$$

Using our earlier framework, it is possible to give another proof of this theorem.

Outline of proof of theorem 6 . Let $u_{+}$enumerate the area of the path below the $x$-axis, and $u_{-}$enumerate the area of the path under the $x$-axis. Then the generating functions $P$ and $B$ of bilateral Dyck paths and Bernoulli random walks satisfy

$$
P\left(z, u_{+}, u_{-}\right)=\frac{1}{1-z^{2}\left(u_{+} D\left(z u_{+}, u_{+}\right)+u_{-} D\left(z u_{-}, u_{-}\right)\right)}
$$

and

$$
B\left(z, u_{+}, u_{-}\right)=P\left(z, u_{+}, u_{-}\right)\left(1+z \sqrt{u_{+}} F\left(z u_{+}, u_{+}\right)+z \sqrt{u_{-}} F\left(z u_{-}, u_{-}\right)\right) .
$$

If we define $Q_{r s}$ such that

$$
\frac{P_{u_{+}^{r} u_{-}^{s}}(z, 1)}{r ! s !} \underset{z \rightarrow \frac{1}{2}}{\sim} \frac{2^{r+s} Q_{r s}}{(1-2 z)^{\frac{3 r+1}{2}}},
$$

and $q(u, v)=\sum Q_{r s}(-u)^{r}(-v)^{s}$, then after differentiating $r$ times for all $r$

$$
\left(1-z^{2}\left(u_{+} D\left(z u_{+}, u_{+}\right)+u_{-} D\left(z u_{-}, u_{-}\right)\right)\right) P\left(z, u_{+}, u_{-}\right)=1
$$

with respect to $u_{+}$and $u_{-}$, setting $u_{+}=u_{-}=1$, and identifying dominant coefficients in $\left(1-4 z^{2}\right)^{-1 / 2}$, we obtain recurrence relations on $\left(Q_{r s}\right)$ which translate to

$$
q\left(u_{-}, u_{+}\right)=-\frac{1}{y\left(u_{-}\right)+y\left(u_{+}\right)}
$$


which can be written as (61) with $u_{+}=\beta \cdot 2^{-1 / 2} \alpha^{-3 / 2}$ and $u_{-}=\gamma \cdot 2^{-1 / 2} \alpha^{-3 / 2}$.

(62) can be proved in the same way, starting with (64).

The equality (67) can be found in exercise 4.18 chapter XIII of [Revuz and Yor (1998)], for more general additive functionals. In the next section, we will show how to establish this equality for functionals which are polynomial in some sense, using only generating functions.

\section{Other functionals}

In [Nguy^en Th^e (2003)], the theorem of Louchard was proved for polynomial functionals using a multivariate version of (4). It is possible to use the same method with (5) to establish a similar theorem, linking standard Brownian motion, meanders and Brownian bridges.

Theorem 7. Let $m$ denote the meander, $b$ denote the Brownian bridge and $W$ the standard Brownian motion. For nonnegative numbers $h_{0}, h_{1}, \ldots, h_{M}$ such that $\left|h_{1}\right|+\cdots+\left|h_{M}\right| \neq 0$, define $h: x \mapsto \sum_{j=0}^{M} h_{j}|x|^{j}$. Let $g_{s}, g_{b}$ and $g_{m}$ denote the double Laplace transforms of the distribution of the functional $\int h(\cdot) d \cdot$ for respectively the standard Brownian motion starting from 0, the Brownian bridge and the meander:

$$
\begin{aligned}
g_{s}(\alpha) & =\int_{0}^{\infty} e^{-\alpha t} \mathbb{E}_{0}\left[e^{-\int_{0}^{t} h\left(W_{s}\right) d s}\right] d t \\
g_{b}(\alpha) & =\int_{0}^{\infty} e^{-\alpha t} \mathbb{E}\left[e^{-\int_{0}^{t} h\left(b_{s}\right) d s}\right] \frac{d t}{\sqrt{2 \pi t}} \\
g_{m}(\alpha) & =\int_{0}^{\infty} e^{-\alpha t} \mathbb{E}\left[e^{-\int_{0}^{t} h\left(m_{s}\right) d s}\right] \frac{d t}{\sqrt{2 \pi t}}
\end{aligned}
$$

Then

$$
g_{s}=2 g_{b} g_{m}
$$

Proof. Fix $M \in \mathbb{N}^{*}$. Let $u_{k}$ enumerate the sum of the $k$-th powers of the absolute value of the altitudes of the path. Then

$$
\left.B\left(z, u_{1}, \cdots, u_{M}\right)=P\left(z, u_{1}, \cdots, u_{M}\right)\left(2 z u_{1} \cdots u_{M}\right) F\left(z u_{1}, \cdots, u_{M}\right)+1\right),
$$

where the $(j+1)$-th component of $F$ is given by

$$
u_{j} u_{j+1}^{j+1} \cdots u_{M-1}^{\left(\begin{array}{c}
M-1 \\
j
\end{array}\right)} u_{M}^{\left(\begin{array}{c}
M \\
j
\end{array}\right)}=\prod_{q=j}^{q=M} u_{q}^{\left(\begin{array}{c}
q \\
j
\end{array}\right)} .
$$

Let $a_{r_{1}, r_{2}, \cdots, r_{M}}^{B}, a_{r_{1}, r_{2}, \cdots, r_{M}}^{P}, a_{r_{1}, r_{2}, \cdots, r_{M}}^{F}$ denote the constants such that, for all $\left(r_{1}, r_{2}, \cdots, r_{M}\right) \in N^{M}$,

$$
\begin{aligned}
& \frac{F_{u_{1} r_{1} u_{2} r_{2} \cdots u_{M}^{r_{M}}(z, 1)}}{r_{1} ! r_{2} ! \cdots r_{M} !} \underset{z \rightarrow \frac{1}{2}}{\sim} \frac{a_{r_{1}, r_{2}, \cdots, r_{M}}^{F}}{(1-2 z)^{\frac{1+\Sigma(2+i) r_{i}}{2}}}, \\
& \frac{P_{u_{1} r_{1}}^{r_{2} \cdots u_{M}^{r_{2}}}(z, 1)}{r_{1} ! r_{2} ! \cdots r_{M} !} \underset{z \rightarrow \frac{1}{2}}{\sim} \frac{a_{r_{1}, r_{2}, \cdots, r_{M}}^{P}}{(1-2 z)^{\frac{1+\Sigma(2+i) r_{i}}{2}}}
\end{aligned}
$$

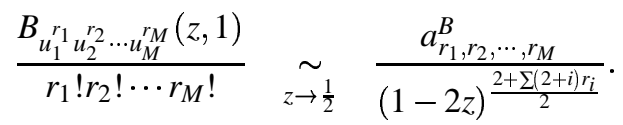


Set

$$
\begin{aligned}
& A_{B}\left(u_{1}, \cdots, u_{M}\right)=\sum a_{r_{1}, r_{2}, \cdots, r_{M}}^{B}\left(-u_{1}\right)^{r_{1}} \cdots\left(-u_{M}\right)^{r_{M}}, \\
& A_{P}\left(u_{1}, \cdots, u_{M}\right)=\sum a_{r_{1}, r_{2}, \cdots, r_{M}}\left(-u_{1}\right)^{r_{1}} \cdots\left(-u_{M}\right)^{r_{M}} \\
& A_{F}\left(u_{1}, \cdots, u_{M}\right)=\sum a_{r_{1}, r_{2}, \cdots, r_{M}}^{F}\left(-u_{1}\right)^{r_{1}} \cdots\left(-u_{M}\right)^{r_{M}} .
\end{aligned}
$$

Then (72) translates into $A_{B}=A_{P} A_{F}$. As we have

$$
\begin{aligned}
& g_{s}(\alpha)=\frac{1}{\alpha} A_{B}\left(\frac{h_{1}}{\alpha^{1+\frac{1}{2}}}, \cdots, \frac{h_{k}}{\alpha^{1+\frac{k}{2}}}, \cdots, \frac{h_{M}}{\alpha^{1+\frac{M}{2}}}\right), \\
& g_{b}(\alpha)=\frac{1}{\sqrt{\alpha}} A_{P}\left(\frac{h_{1}}{\alpha^{1+\frac{1}{2}}}, \cdots, \frac{h_{M}}{\alpha^{1+\frac{M}{2}}}\right), \\
& g_{m}(\alpha)=\frac{1}{2 \sqrt{\alpha}} A_{F}\left(\frac{h_{1}}{\alpha^{1+\frac{1}{2}}}, \cdots, \frac{h_{M}}{\alpha^{1+\frac{M}{2}}}\right),
\end{aligned}
$$

this proves the theorem for $h_{0}=0$. For $h_{0} \neq 0$, just replace $\alpha$ with $\alpha+h_{0}$.

This result remains valid for Borel functionals, and was already shown in [Revuz and Yor (1998); Yor (1995)], using the Brownian comeander

$$
\tilde{m}_{u}=\frac{1}{1-g_{1}}\left|B_{g_{1}+u\left(1-g_{1}\right)}\right|,
$$

which is independent from what happened before $g_{1}$, and using, in the continuous world, a decomposition which is similar to the combinatorial decomposition used here: a Brownian motion of duration $g_{1}$ (time of the last return to the $x$-axis before the instant 1), followed by the comeander (83).

For the quadratic functional with $h(x)=x^{2}$, injecting the known results (via continuous objects)

$$
\begin{aligned}
\mathbb{E}_{0}\left[e^{-\int_{0}^{t} W_{s}^{2} d s}\right] & =(\cosh (t \sqrt{2}))^{-1 / 2}, \\
\mathbb{E}\left[e^{-\int_{0}^{t} b_{s}^{2} d s}\right] & =(t \sqrt{2} / \sinh (t \sqrt{2}))^{1 / 2}, \\
\mathbb{E}\left[e^{-\int_{0}^{t} m_{s}^{2} d s}\right] & =(\cosh (t \sqrt{2}))^{-1}(t \sqrt{2} / \sinh (t \sqrt{2}))^{1 / 2},
\end{aligned}
$$

in (71), and inverting in $\alpha$ the Laplace transforms on each side of the obtained equation, gives back the convolution identity

$$
\frac{1}{(\cosh v)^{1 / 2}}=\frac{1}{\pi} \int_{0}^{v} \frac{d x}{(\sinh x)^{1 / 2}(\sinh (v-x))^{1 / 2} \cosh (v-x)}
$$

already discussed in [Jeanblanc et al. (1997)].

Using a proof similar to the one of Theorem 7, we could show generalized versions of (61) (or (67)) and (62) valid for polynomial functionals. 


\section{Conclusion}

The use of generating functions gives another approach for finding several results on the distribution of the area of Brownian motion, with no more prerequisites than singularity analysis, and provides some insight into the relations between the different types of distribution. Though the method of moments does not seem to provide new results it can be applied to combinatorial objects that do not necessarily converge to a known Brownian motion. For other functionals it seems difficult to avoid the use of catalytic variables (like $u_{1}, \cdots, u_{M-1}$ for functionals with $h(x)=x^{M}$ ) and get the limit distribution.

Possible future work includes study of the joint distribution of the area and the final altitude of meanders, as given in [Cifarelli (1975)] for Brownian motion, using a functional equation of the type [Flajolet and Sedgewick (2001)]

$$
F(z, u, v)=1+z\left(u v+\frac{1}{u v}\right) F(z, u, v u)-\frac{1}{u v} F(z, u, 0),
$$

where $F$ is the generating function of meanders enumerated by size (variable $z$ ), area (variable $u$ ), and final altitude (variable $v$ ). For this type of equation moments might not be sufficient.

Other papers of interest are for example [Gutjahr and Pflug (1992), Gittenberger (1999)] which show that the contour process of random trees converges towards a Brownian excursion. The different types of Brownian motion studied here should appear as limits of a much greater family of discrete random walks than the ones that are studied here.

\section{References}

M. Abramowitz and I. A. Stegun. Handbook of Mathematical Functions. Dover, 1973. A reprint of the tenth National Bureau of Standards edition, 1964.

P. Biane and M. Yor. Valeurs principales associées au temps locaux Browniens. Bull. Sci. Math. (2), 111: 23-101, 1987.

D. Cifarelli. Contributi intorno ad un test per l'omogeneità tra due campioni. G. Econom. Ann. Econ. (N.S.), 34:233-249, 1975.

M. Drmota. Stochastic analysis of tree-like data structures. Special edition of the Royal Society on Stochastic Analysis, 2003.

W. Feller. An Introduction to Probability Theory and Its Applications, volume 2. John Wiley, 1971.

P. Flajolet and R. Sedgewick. The average case analysis of algorithms: Complex asymptotics and generating functions. Research Report 2026, INRIA, 1993. 100 pages.

P. Flajolet and R. Sedgewick. Functional equations, rational and algebraic functions. Research Report 4103, INRIA, 2001. 98 pages.

B. Gittenberger. On the contour of random trees. SIAM Journal on Discrete Mathematics, 12(4):434-458, 1999.

W. Gutjahr and G. C. Pflug. The asymptotic contour process of a binary tree is a brownian excursion. Stochastic Processes and their Applications, 41:69-89, 1992. 
K. Ito and H. McKean. Diffusion processes and their sample paths. Academic Press, New York, 1965.

M. Jeanblanc, J. Pitman, and M. Yor. The Feynman-Kac formula and decomposition of Brownian paths. Computational and Applied Mathematics, 16:27-52, 1997.

M. Kac. On the distribution of certain Wiener functionals. Transactions of the American Mathematical Society, 65:1-13, 1949.

G. Louchard. Kac's Formula, Lévy's Local Time and Brownian Excursion. Journal of Applied Probability, 21:479-499, 1984.

M. Nguy^en Th^e. Area and Inertial Moment of Dyck Paths. Combinatorics, Probability and Computing, 2003. Submitted.

M. Perman and J. Wellner. On the distribution of Brownian areas. Annals of Applied Probability, 6: 1091-1111, 1995.

D. Revuz and M. Yor. Continuous Martingales and Brownian Motion, 3rd edition. Springer-Verlag, New York, 1998.

L. A. Shepp. On the Integral of the Absolute Value of the Pinned Wiener Process. The Annals of Probability, 10:234-239, 1982.

R. P. Stanley. Enumerative Combinatorics, volume 2. Cambridge University Press, New York, 1999.

L. Takács. A Bernoulli Excursion and its Various Applications. Advances of Applied Probability, 23: 557-585, 1991a.

L. Takács. On a probability problem connected with railway traffic. Journal of Applied Mathematics and Stochastic Analysis, 4(1):1-27, $1991 \mathrm{~b}$.

L. Takács. On the distribution of the integral of the absolute value of the Brownian motion. Annals of Applied Probability, 3:186-197, 1993.

L. Takács. On the total heights of random rooted binary trees. Journal of Combinatorial Theory, Series B, 61(2):155-166, 1994.

L. Tolmatz. Asymptotics of the distribution of the integral of the absolute value of the Brownian bridge for large arguments. The Annals of Probability, 28:132-139, 2000.

L. Tolmatz. Asymptotics of the distribution of the integral of the positive part of the Brownian bridge for large arguments. 2001. http://www.tolmatz . net/PAPERS/04POSBB/30s.pdf.

M. Yor. Local times and excursions for brownian motion: a concise introduction. Postgrado de Matemáticas, Facultad de Ciencias, Universidad Central de Venezuala, Caracas, 1995. Lecciones en Mathemáticas, vol. 1. 